\title{
The mysterious spin gap in high-temperature superconductors: new
} NMR/NQR studies

\author{
Brinkmann, D
}

\begin{abstract}
We review work, done mainly at the author's laboratory, on the spin gap which is the NMR manifestation of the pseudogap observed in the normal state of high-temperature superconductors. The relation of the spin gap to an electronic crossover in $\mathrm{YBa} 2 \mathrm{Cu} 4 \mathrm{O} 8$ is discussed. A possible explanation of both effects by assuming a charge density wave transition is presented. This suggestion is supported by measuring the isotope dependence of the spin gap in $\mathrm{YBa} 2 \mathrm{Cu} 4 \mathrm{O} 8$
\end{abstract}

DOI: https://doi.org/10.1007/bf03161871

Posted at the Zurich Open Repository and Archive, University of Zurich

ZORA URL: https://doi.org/10.5167/uzh-155880

Journal Article

Published Version

Originally published at:

Brinkmann, D (1998). The mysterious spin gap in high-temperature superconductors: new NMR/NQR studies. Applied Magnetic Resonance, 15(2):197-202.

DOI: https://doi.org/10.1007/bf03161871 


\title{
The Mysterious Spin Gap in High-Temperature Superconductors: New NMR/NQR Studies*
}

\author{
D. Brinkmann \\ Physik-Institut, Universität Zürich, Zürich, Switzerland
}

Received August 2, 1998

\begin{abstract}
We review work, done mainly at the author's laboratory, on the spin gap which is the NMR manifestation of the pseudogap observed in the normal state of high-temperature superconductors. The relation of the spin gap to an electronic crossover in $\mathrm{YBa}_{2} \mathrm{Cu}_{4} \mathrm{O}_{8}$ is discussed. A possible explanation of both effects by assuming a charge density wave transition is presented. This suggestion is supported by measuring the isotope dependence of the spin gap in $\mathrm{YBa}_{2} \mathrm{Cu}_{4} \mathrm{O}_{8}$.
\end{abstract}

\section{Introduction}

The mechanism of high-temperature superconductivity still remains under debate. It is commonly agreed that an understanding of the normal state of these materials is a prerequisite for the elucidation of the superconductivity mechanism. A typical question, not yet satisfactorily answered, concerns the origin of the so-called pseudogap in underdoped cuprate superconductors [1]. Evidence for the presence of a gap-like structure in the electronic excitation spectra below a certain temperature $T^{*}$, which lies above $T_{c}$, has been found by many techniques like NMR, neutron scattering, infrared conductivity, specific heat, thermoelectric power. Recently, angular resolved photoemission spectroscopy (ARPES) has provided direct evidence for the existence, above $T_{c}$, of a pseudogap in the density of states at the Fermi level $[2,3]$ and tunneling spectroscopy [4] has shown that the pseudogap is also present in overdoped samples. In NMR and neutron scattering the pseudogap reveals itself as a spin gap. In this paper, we will review some NMR/NQR studies, mostly from our laboratory, which are concerned with the spin gap and its possible relation to other gaps like the electronic gap.

\footnotetext{
- Presented at the Joint 29th AMPERE and 13th ISMAR International Conference on Magnetic Resonance and Related Phenomena, Berlin, August 2-7, 1998
} 


\section{The Spin Gap Effect}

After the discovery of the spin gap effect in $\mathrm{YBa}_{2} \mathrm{Cu}_{4} \mathrm{O}_{7-\delta}$ by neutron scattering [5], several NMR groups have regarded the spin gap effect to be responsible for the peculiar temperature variation of the spin-lattice relaxation time, $T_{1}$, at least in the normal conducting state [6]. The occurrence of a spin gap means that spectral weight in the electron spin fluctuations is transferred from lower to higher energy. The effect competes with the antiferromagnetic (AF) fluctuations which increase with descending temperature. Below a temperature $T^{*}$, where the maximum of the "spin-lattice relaxation rate per temperature unit", $\left(T_{1} T\right)^{-1}$, occurs, the spin-gap effect predominates and thus $\left(T_{1} T\right)^{-1}$ is decreasing. In underdoped structures, $T^{*}$ lies well above $T_{\mathrm{c}}$ while in overdoped compounds $T^{*}$ nearly coincides with $T_{\mathrm{c}}$. For $\mathrm{YBa}_{2} \mathrm{Cu}_{4} \mathrm{O}_{8}$, which we have studied intensively, the corresponding values are $T_{\mathrm{c}}=81 \mathrm{~K}$ and $T^{*} \approx 150 \mathrm{~K}$.

Since at present no theoretical derivation of $T_{1}$ exists which takes into account the presence of the spin gap, it became customary to analyze $\left(T_{1} T\right)^{-1}$ data with the help of the following phenomenological function $[6,7]$ :

$$
\left(T_{1} T\right)^{-1}=C T^{-\alpha}\left(1-\tanh ^{2}\left(\frac{\Delta}{2 T}\right)\right) .
$$

It may be derived from a function used by Tranquada et al. [8] to fit properly the dynamic susceptibility data in the presence of a spin gap, as determined by neutron scattering measurements.

In Eq. (1), the hyperbolic tangent describes the temperature-dependent gap and the factor $C T^{-\alpha}$, with $\alpha \simeq 1$, takes into account the high-temperature Curie-like divergence of $\left(T_{1} T\right)^{-1} . \Delta$ is a measure for the gap; it is proportional to $T^{*}$, de-

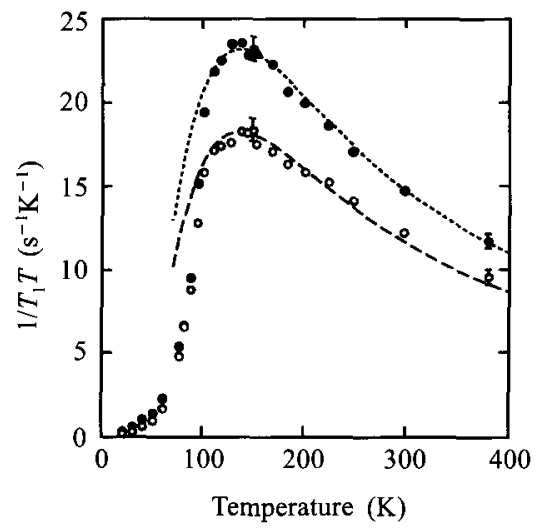

Fig. 1. Temperature dependence of $1 / T_{1} T$ of the planar $\mathrm{Cu}(2)(\bullet)$ and $\mathrm{Cu}(3)(0)$ in $\mathrm{Y}_{2} \mathrm{Ba}_{4} \mathrm{Cu}_{7} \mathrm{O}_{15}$. The lines are the fits of Eq. (1). From [9]. 
fined as the maximum of $\left(T_{1} T\right)^{-1}$ as defined by Eq. (1). So, both, $\Delta$ and $T^{*}$ can be used as a proper parameter for the temperature scale of the spin gap.

As an example for applying Eq. (1), we mention $\mathrm{Y}_{2} \mathrm{Ba}_{4} \mathrm{Cu}_{7} \mathrm{O}_{15}$ which is a "mixedlayer" compound with the individual layers of the $\mathrm{CuO}_{2}$ bilayer containing two inequivalent $\mathrm{Cu}$ sites, $\mathrm{Cu}(2)$ and $\mathrm{Cu}(3)$. The fit of Eq. (1) to the data of both sites (see Fig. 1) yields $\Delta=240 \pm 20 \mathrm{~K}$ and $\alpha=1.25$ [9]; these values agree, within the error limits, with $\Delta=260 \pm 10 \mathrm{~K}$ and $\alpha=1.25$ we obtained for our $\mathrm{Cu}(2)$ data in $\mathrm{YBa}_{2} \mathrm{Cu}_{4} \mathrm{O}_{8}$.

Without attempting to discuss the many theoretical scenarios and models of hightemperature superconductivity in which the appearance of the pseudogap is an essential ingredient [10-12], we will discuss, in the next section, an experimental study which opened an alternative way to the understanding of the origin of the spin gap.

\section{Electronic Crossover in $\mathrm{YBa}_{2} \mathrm{Cu}_{4} \mathrm{O}_{8}$}

A detailed NMR/NQR study of $\mathrm{YBa}_{2} \mathrm{Cu}_{4} \mathrm{O}_{8}$ [13] has revealed that an electronic crossover takes place in this compound at a temperature $T^{\dagger} \approx 180 \mathrm{~K}$. This conclusion is based on the fact that the temperature dependence of several NMR/NQR parameters exhibits an anomaly around $T^{\dagger}$. For instance, the $\mathrm{Cu}(2) \mathrm{NQR}$ frequency, ${ }^{63} v_{\mathrm{Q}}$, exhibits a minimum; the $\mathrm{Cu}(2)$ total magnetic shift, ${ }^{65} K_{\mathrm{c}}$, has a kink; the chain copper, $\mathrm{Cu}(1), \mathrm{NQR}$ frequency and NMR relaxation rate show a change in slope. Further anomalies were found in the chain oxygen, O(4), NMR relaxation and in the ratio of the plane oxygen and yttrium relaxation rates, ${ }^{17} W_{c}{ }^{89} W_{c}$, (where $c$ denotes the direction of the magnetic field).

The analysis of these data led us to the conclusion that an electronic crossover rather than a structural phase transition (neither first nor second order) occurs at

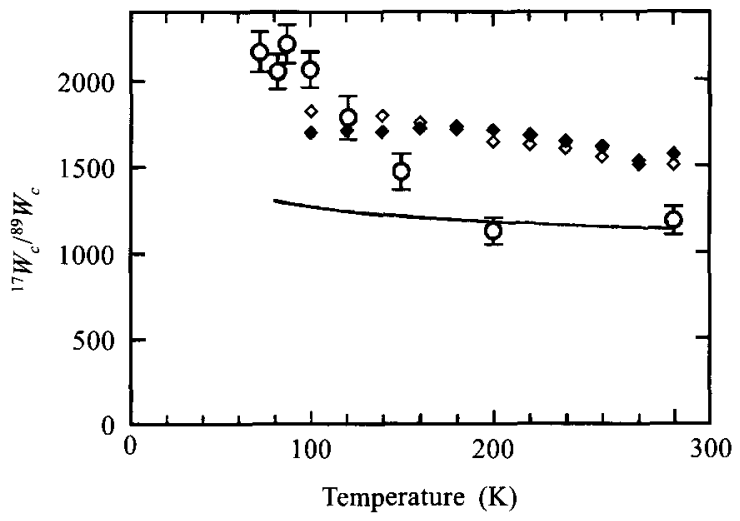

Fig. 2. ${ }^{17} W_{c} /{ }^{89} W_{c}$ vs. temperature. $\bigcirc \mathrm{YBa}_{2} \mathrm{Cu}_{4} \mathrm{O}_{8}[13], \diamond \mathrm{YBa}_{2} \mathrm{Cu}_{3} \mathrm{O}_{6.63}[14], \diamond \mathrm{YBa}_{2} \mathrm{Cu}_{3} \mathrm{O}_{7}$ [14]. The solid line is a calculation (see text). 
$T^{\dagger}$. This crossover involves a charge redistribution, namely a transfer of holes from the chains into the planes. The enhanced charge fluctuations in the chain and the plane, below $T^{\dagger}$, cause an increase of ${ }^{17} W_{c}{ }^{89} W_{c}$ for decreasing temperatures (see Fig. 2), since only ${ }^{17} W_{c}$ is enhanced while yttrium, because of its spin $1 / 2$, cannot couple to the charge fluctuations.

In contrast to this explanation, the solid line in Fig. 2 fails to describe the temperature dependence of ${ }^{17} W_{c}{ }^{89} W_{c}$. The line has been calculated following [15] where relaxation is treated within the phenomenological Millis-Monien-Pines (MMP) model [16] by assuming additional next-nearest neighbor coupling and incommensurate AF fluctuations. The MMP model includes, in addition to the normal Fermi-liquid contribution to the relaxation, strong AF fluctuations which lead to the anomalous behavior of the spin-lattice relaxation in high-temperature superconductors.

What is the origin of the crossover? A tentative mechanism will be proposed in the next section.

\section{Relation to Charge Density Waves}

It is well known that a quasi two-dimensional metal with a strong anisotropic Fermi surface is unstable with respect to a charge density wave (CDW) transition [17]. Since Fermi surfaces in layered cuprates, according to photoemission data [18], are really different from a perfect circle or cylinder, it is tempting to analyze the CDW scenario as a possible origin for the opening of a pseudogap in the normal state of these compounds. Although this point has been emphasized by many authors, however, to our knowledge, numerical calculations have not yet been performed. In this context, the electronic crossover shows itself in a new light: does the occurrence of this crossover support the idea [19] that the spin gap phenomenon is caused, at least partly, by a transition due to a CDW?

Eremin et al. have studied this problem [20]. Starting from the $t-J$ model and including electron-phonon interaction, they derived the gap equation for a $\mathrm{CDW}$ in the $\mathrm{CuO}_{2}$ plane, using the singlet correlated band in the normal state. The model not only allowed us to explain the important features of the momentum and temperature dependences of the pseudogap, it also could describe the strong temperature dependence of the $\mathrm{Cu}(2)$ magnetic shift ${ }^{65} K_{a b}\left(\mathrm{~B}_{0} \perp c\right)$ in $\mathrm{YBa}_{2} \mathrm{Cu}_{4} \mathrm{O}_{8}$; the shift depends on the spin susceptibility and had been measured previously [21].

A severe test, whether the CDW approach is correct, is the prediction of a dependence of $T^{*}$ on the isotope mass. The experimental verification of this prediction will be discussed in the next section.

\section{Isotope Effect of the Spin Gap}

We have determined the isotope dependence of $T^{*}$ in $\mathrm{YBa}_{2} \mathrm{Cu}_{4} \mathrm{O}_{8}$ in the following way [22]. Two oxygen exchanged samples $\left({ }^{16} \mathrm{O}\right.$ and $\left.{ }^{18} \mathrm{O}\right)$ were inserted into the 
probe head with two radiofrequency coils which allowed an automatic switch over of the electronics from one sample to the other. Among others, we measured the temperature dependence of the ${ }^{63} \mathrm{Cu} \mathrm{NQR} T_{1}$ relaxation time with a precision of $0.3 \%$ for the $\left(T_{1} T\right)^{-1}$ data. To our knowledge, this is the highest accuracy of all measurements of this type.

The $\left(T_{1} T\right)^{-1}$ data were fitted with a slightly modified version of Eq. (1). The best value for the $T^{*}$ shift is $0.96 \mathrm{~K}$ resulting in an isotope exponent $\alpha_{T^{*}}=0.061(8)$ which is defined by $\alpha_{T^{*}}=-\Delta \ln \left(T^{*}\right) / \Delta \ln (m)$. The corresponding value for $T_{\mathrm{c}}$ measured in the same samples, is $\alpha_{T_{\mathrm{c}}}=0.056(12)$. The agreement of both values within the error bars suggests a common origin for the superconducting and the spin gap.

Our result contrasts with a recent measurement of the ${ }^{89} \mathrm{Y}$ NMR Knight shift which provided no evidence of an isotope effect on the spin gap [24]. A possible explanation for this discrepancy is that one must distinguish between the behavior of the spin susceptibility at wavevector zero, as probed by the Knight shift, and at the antiferromagnetic wavevector, probed by spin-lattice relaxation.

The first calculation of the isotope dependence of the pseudogap, performed within the $\mathrm{CDW}$ scenario and assuming $T_{\mathrm{CDW}}=180 \mathrm{~K}$ [21], resulted in $\alpha_{T_{\mathrm{CDW}}} \approx$ 0.17 which is larger than the experimental value for $\alpha_{T^{*}}$. Subsequent improvements of the calculation [25] by taking into account also superexchange interaction of the copper spins and Coulomb repulsion between holes residing at neighboring sites yielded $\alpha_{T_{\mathrm{CDW}}}=0.028$ which is roughly a factor of 2 smaller than the experimental value.

Although $\alpha_{T_{\mathrm{CDW}}}$ and $\alpha_{T^{*}}$ do not need to be identical, the fact that both exponents are of the same order, suggests a close relation between the CDW transition and the opening of the spin gap. It remains to be shown whether both effects are a manifestation of a common underlying mechanism.

\section{Summary}

While the presence of the spin-gap effect in NMR is known for quite some time, its origin still remains controversial. Our recent detection of an electronic crossover in $\mathrm{YBa}_{2} \mathrm{Cu}_{4} \mathrm{O}_{8}$, taking place close to the temperature where the spin gap opens, has offered a new approach to solve this problem. The occurrence of the crossover supports the idea that the spin-gap phenomenon is caused, at least partly, by a transition due to a charge density wave (CDW). Within this framework, we have calculated important features of the CDW gap and we could explain the temperature dependence of the planar $\mathrm{Cu}$ Knight shift in $\mathrm{YBa}_{2} \mathrm{Cu}_{4} \mathrm{O}_{8}$. In particular, we calculated the coefficient of the isotope effect on the $\mathrm{CDW}$ gap. This value agrees fairly well with our experimental determination of the spin gap isotope coefficient. Since the latter value also agrees with the $T_{\mathrm{c}}$ isotope coefficient (all measured in the same sample), it is very tempting to suggest that all three gaps are related to each other. 


\section{References}

[1] Service R.F.: Science 278, 1880 (1997)

[2] Loeser A.G., Shen Z.-X., Dessau D.S., Marshall D.S., Park C.H., Fournier P., Kapitulnik A.: Science 273, 325 (1996)

[3] Ding H., Yokoya T., Campuzano J.C., Takahashi T., Randeria M., Norman M.R., Mochiku T., Hadowaki K., Giapintzakis J.: Nature 382, 51 (1996)

[4] Renner Ch., Revaz B., Genoud J.-Y., Kadowaki K., Fischer Ø.: Phys. Rev. Lett. 80, 149 (1998)

[5] Rossat-Mignod J., Regnault L.P., Vettier C., Burlet C., Henry J.Y., Lapertod G.: Physica B 169, $58(1991)$

[6] Brinkmann D., Mali M. in: NMR - Basic Principles and Progress (Diehl P., Fluck E., Günther H., Kosfeld R., Seelig J., eds.), vol. 31, p. 171. Berlin: Springer 1994.

[7] Mehring M.: Appl. Magn. Reson. 3, 423 (1992)

[8] Tranquada J.M., Gehring P.M., Shirane G., Shamoto S., Sato M.: Phys. Rev. B 46, 5561 (1992)

[9] Stern R., Mali M., Mangelschots I., Roos J,, Brinkmann D., Heinmaa I., Genoud J.-Y., Graf T., Muller J.: Phys. Rev. B 50, 426 (1994)

[10] Pines D.: Physica C 282-287, 273 (1997)

[11] Fukuyama H., Kohno H., Tanamoto T.: Physica C 235-240, 63 (1994)

[12] Markiewicz R.S.: J. Phys. Chem. Solids 56, 1637 (1995); Emery V.J., Kivelson S.A.: Phys. Rev. B 56, 6120 (1997); Alexandrov A.S., Mott N.F.: Rep. Prog. Phys. 57, 1197 (1994)

[13] Suter A., Mali M., Roos J., Brinkmann D., Karpinski J., Kaldis E.: Phys. Rev. B 56, 5542 (1997)

[14] Takigawa M., Hults W.L., Smith J.L.: Phys. Rev. Lett. 71, 2650 (1993)

[15] Zha Y., Barzykin V., Pines D.: Phys. Rev. B 54, 7561 (1996)

[16] Millis A.J., Monien H.: Phys. Rev. B 45, 3059 (1992)

[17] Gruner G.: Density Waves in Solids. New York: Addison-Wesley 1994.

[18] Shen Z.-X., Dessau D.S.: Phys. Rep. 253, 1 (1995)

[19] Markiewicz R.S.: Physics C 193, 323 (1992)

[20] Eremin I., Eremin M., Varlamov S., Brinkmann D., Mali M., Roos J.: Phys. Rev. B 56, 11305 (1997)

[21] Bankay M., Mali M., Roos J., Brinkmann D.: Phys. Rev. B 50, 6416 (1994)

[22] Raffa F., Ohno T., Mali M., Roos J., Brinkmann D., Conder K., Eremin M.: Phys. Rev. Lett. (in press)

[23] Williams G.V.M., Tallon J.L., Quilty J.W., Trodahl H.J., Flower N.E.: Phys. Rev. Lett. 80, 377 (1998)

[24] Varlamov S.V., Eremin M.V., Eremin I.M.: JETP Lett. 66, 569 (1997)

Author's address: Prof. Dr. Detlef Brinkmann, Physik-Institut, Universität Zürich, Winterthurerstrasse 190, CH-8057 Zürich, Switzerland 